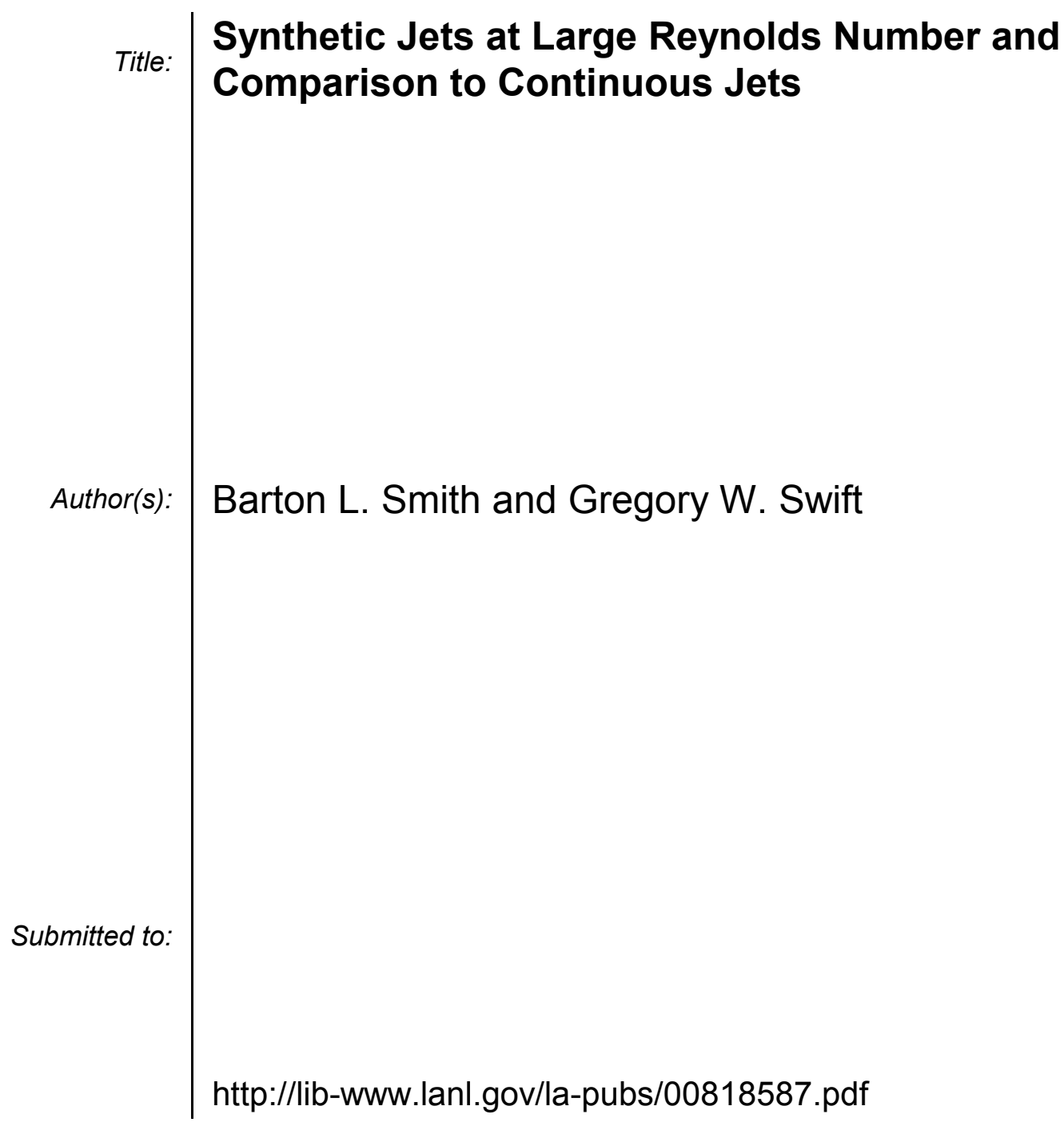

Los Alamos National Laboratory, an affirmative action/equal opportunity employer, is operated by the University of California for the U.S. Department of Energy under contract W-7405-ENG-36. By acceptance of this article, the publisher recognizes that the U.S. Government retains a nonexclusive, royaltyfree license to publish or reproduce the published form of this contribution, or to allow others to do so, for U.S. Government purposes. Los Alamos National Laboratory requests that the publisher identify this article as work performed under the auspices of the U.S. Department of Energy. Los Alamos National Laboratory strongly supports academic freedom and a researcher's right to publish; as an institution, however, the Laboratory does not endorse the viewpoint of a publication or guarantee its technical correctness. 


\title{
Synthetic Jets at Large Reynolds Number and Comparison to Continuous Jets *
}

\author{
Barton L. Smith ${ }^{\dagger}$ and Gregory W. Swift ${ }^{\ddagger}$ \\ Condensed Matter and Thermal Physics Group \\ Los Alamos National Laboratory
}

\begin{abstract}
:
Experimental measurements and flow visualization of synthetic jets and similar continuous jets are described. The dimensionless stroke length necessary to form a 2-D synthetic jet is between 5 and 10, with wider-nozzle jets consistently requiring a smaller value. Synthetic jets are wider, slower and have more momentum than similar continuous jets. Synthetic jets are generated using four nozzle widths that vary by a factor of four, and the driving frequency is varied over an order of magnitude. The resultant jets are in the range $13.5<L_{o} / h<80.8$ and $695<R e_{U_{o}}<14700$. In spite of the large range of stroke lengths, the near-field behavior of the synthetic jets scales with $L_{o} / h$.
\end{abstract}

*Copyright (C)2001 by Barton Smith. Published by the American Institute of Aeronautics and Astronautics Inc., with permission.

${ }^{\dagger}$ Postdoctoral researcher, AIAA member

$\ddagger$ Technical Staff Member

American Institute of Aeronautics and Astronautics 


\section{INTRODUCTION}

The synthetic jet is a mean fluid motion generated by high-amplitude oscillatory flow through an orifice or nozzle. Since its first use in 1994 [1], the synthetic jet has become a popular laboratory flow-control actuator. The primary advantage of the synthetic jet is its zero-net-mass nature, which eliminates the need for plumbing, and, when applied to a base flow, results in unique effects not possible with steady or pulsed suction or blowing. These effects include the creation of closed recirculation regions $[2,3]$ and low pressure regions $[1,4,3]$, and the introduction of arbitrary scales to the base flow $[2,4]$. Low-Reynoldsnumber synthetic jets similar to the devices used in recent flow-control studies have been studied extensively, both experimentally [5] and numerically $[6,7]$. If synthetic jets are to move from the laboratory to flight hardware the Reynolds numbers must be much larger. No literature known to the authors exists on 2-D synthetic jets of Reynolds numbers greater than 2000 .

Although some limited information exists about the stroke required to form an axisymmetric synthetic jet $[8,11]$, no similar information exists for 2-D synthetic jets, nor is it known how this formation threshold varies with other parameters. In a study on nonlinear impedance of a round orifice, Ingard [8] noted that a jet is formed for sufficiently high oscillatory velocity, and that the necessary velocity increases with frequency. Below this threshold, turbulent motions were observed, which were likely due to vortices being generated and then reingested. At even lower amplitudes, motions correctly described as acoustic streaming were observed. (Since that time, many other acousticians have described the jetting motion of a synthetic jet as acoustic streaming. However, since the mean motions induced by a synthetic jet are of the same order as the oscillatory motions, this is not acoustic streaming, for which mean motions occur only at second order.)

Another obvious and as of yet not entirely answered question is how synthetic jets compare to continuous jets at the same Reynolds number. Smith and Glezer [5] showed that a synthetic jet with $R e_{U_{o}}=$ 383 has many characteristics which are similar to continuous higher-Reynolds-number jets. A direct comparison was not possible, since data on continuous jets with $R e_{h}<1000$ are scarce.

The purpose of this study is three fold: 1) to determine the necessary non-dimensional stroke length necessary for jet formation, 2) to learn more about how synthetic jets are similar to and different from continuous jets, and which velocity scale of the synthetic jet should be matched with the average velocity of a continuous jet to get a similar flow, 3) to investigate the effects of the dimensionless parameters $L_{o} / h$ and $R e_{U_{o}}$ separately using phase-locked flow visualization and exit plane profiles, spreading rates and velocity spectra, and to extend the results of Ref. [5] to much larger Reynolds number.

The large Reynolds numbers necessary for this study are achieved in an oscillatory flow facility we have recently built at Los Alamos National Laboratory. The rectangular jet nozzle, which is identical for the continuous and the synthetic jets, is formed by two 24.1-cm long blocks which can be moved in the cross-stream direction to adjust the nozzle width (Fig. 1). The facility is filled with air at Los Alamos atmospheric pressure $(78.6 \mathrm{kPa})$.

For this study, the cross-stream nozzle width $h$ is varied between $0.51 \mathrm{~cm}$ and $2.0 \mathrm{~cm}$, while the spanwise dimension is fixed at $15.2 \mathrm{~cm}$, resulting in an aspect ratio of 30 for the smallest value of $h$. Both ends of the blocks have a $0.64-\mathrm{cm}$ radius to prevent flow separation during inflow in the oscillatory cases. The origin of the $x$ axis is taken as the beginning of the exit radius rather than the exit plane, since flow visualization shows that the rollup of the vortex pairs begins there for most of the synthetic jets. Glass walls at the span-wise edges of the jet extend the full length of the measurement domain to help maintain a 2-D flow. The widely spaced cross-stream side walls, which are necessary downstream of the nozzle for structural reasons, are $20 \mathrm{~cm}$ apart and are perforated to allow fluid to pass through relatively unimpeded.

Oscillations are generated by a set of eight JBL loudspeakers, nominally rated at 600 Watts, attached to a plenum below the nozzle. The driver system is described in detail in Ref. [9]. The use of loudspeakers allows for a more continuous range of driving frequencies than has been achieved in the past. The facility can generate oscillating velocity amplitudes up to 50 $\mathrm{m} / \mathrm{s}$ in the frequency range $10<f<100 \mathrm{~Hz}$.

A continuous jet is formed by switching off the 
speakers and blowing compressed air into the plenum. Hot-wire measurements of the continuous jets indicate that the flow at the exit is symmetric, nearly fully developed, and has a fluctuation level less than $5 \%$ of the centerline mean.

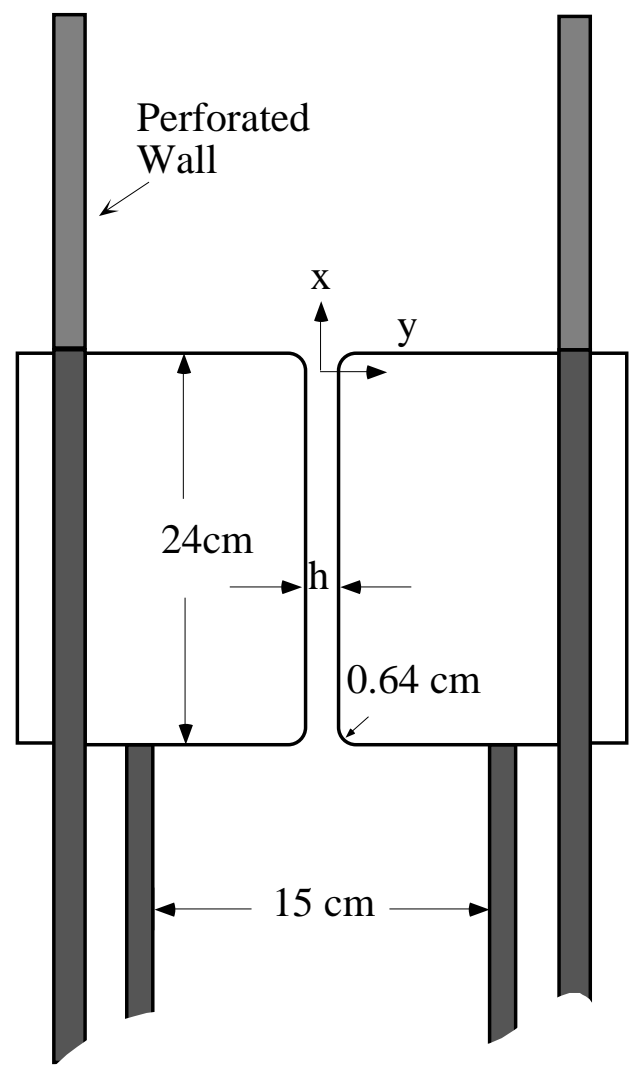

Figure 1: Schematic of the apparatus used in this study. Pressure oscillations are produced by a set of drivers below the nozzle. The nozzle blocks extend $15.2 \mathrm{~cm}$ into the page. The top of the facility is open to local atmospheric pressure $(78.6 \mathrm{kPa})$

Velocity measurements are made using a single straight hot wire, centered spanwise and traversed in the cross-stream and axial directions. Since the sensor is not sensitive to flow direction, measurements are limited to regions of small cross-stream velocity. All measurements are made phase-locked to the driving waveform, and are phase averaged over more than
100 cycles. Velocities are triply decomposed: We assume that the velocity takes the form $\tilde{u}=U+\hat{u}+u^{\prime}$, where $U$ is the time-averaged velocity, $\hat{u}$ is the phaseaveraged time-dependent value and $u^{\prime}$ is the cycleto-cycle fluctuation. Phase-average results reported herein are the phase-averaged value plus the mean, or $u=U+\hat{u}$. When flow reversals are known to be present, such as near the exit plane of a synthetic jet, the velocity traces are derectified before phase averaging is performed.

In order to facilitate a comparison between a synthetic jet and a continuous jet, we need to choose a velocity scale for a synthetic jet, which has a zero mean flow rate at the exit plane. Smith and Glezer [5] proposed the use of the velocity scale

$$
U_{o}=L_{o} f=f \int_{0}^{T / 2} u_{o}(t) d t .
$$

where $u_{o}(t)$ is the centerline nozzle velocity (we will use the cross-stream average, see below), $T=1 / f$ is the oscillation period, and $L_{o}$ (stroke length) is the length of the slug of fluid pushed from the nozzle during the blowing stroke. (In general, time-averaged velocities will be capitalized in this paper.) While other workers have used the maximum spatial-averaged velocity $u_{\max }$ (which is $\pi$ times $U_{o}$ for purely sinusoidal oscillations) or the rms velocity at the exit plane as the velocity scale, Smith and Glezer [5] argued for use of $U_{o}$ since continuous jets with $U_{\text {ave }}=U_{o}$ have the same volume flux directed downstream averaged over a cycle at the exit plane. Unlike the millimeter-scale orifice used in Ref. [5], the larger nozzle in the current work allows for complete phase-locked velocity profiles at the exit plane. For this nozzle, the outward flow resembles oscillatory pipe flow [10] while the inward flow is much more slug-like. Therefore $u_{o}(t)$ will be defined herein as the cross-stream averaged exit-plane velocity.

For the synthetic jets, the Reynolds number is defined as $R e_{U_{o}}=U_{o} h / \nu$, where $\nu$ is the kinematic viscosity. In the continuous jets, the crossstream averaged velocity, $U_{\text {ave }}$, is used as the velocity scale and the Reynolds number is defined as $R e_{h}=U_{\text {ave }} h / \nu$. Continuous and synthetic jets with matched Reynolds numbers based on these definitions will be referred to as "similar" herein.

For synthetic jets created by a sinusoidal slug flow, two independent dimensionless parameters com- 
pletely describe the jet. Although many choices are possible, we will use the dimensionless stroke length $L_{o} / h$ and the Reynolds number defined above.

Hot-wire velocity data are complimented by schlieren photographs that are taken phase-locked to the driving signal. A small amount of a heavy gas (tetrafluoroethane) is introduced below the nozzle blocks before the image is acquired to create the necessary gradients in the index of refraction.

The remainder of this paper is divided into three sections. In the first, we will discuss the value of $L_{o} / h$ necessary for formation of a synthetic jet. Second, we will compare synthetic jets to conventional jets. Finally, the effects of $L_{o} / h$ and $R e_{U_{o}}$ on synthetic jet behavior will be considered.

\section{SYNTHETIC JET FORMA- TION PARAMETERS}

For an axisymmetric orifice of diameter $D$, Ingard [8] and Smith et al. [11] showed that a synthetic jet forms when $L_{o} / D>1$. Below this level, a vortex ring may form, but is ingested during the suction stroke. To our knowledge, no such criterion has been published for 2-D synthetic jets.

A synthetic jet is formed when each vortex pair that is ejected during the blowing stroke propagates downstream with sufficient speed to be out of the influence of the sink-like flow during the suction stroke. If one assumes that the flow behaves potentially within the formation domain, then it is reasonable to model the suction stroke of the synthetic jet by the superposition of a sink at the exit and a counterrotating vortex pair at some distance downstream. If one simplistically assumes that a jet is formed when the velocity induced at each vortex by its neighbor is greater than or equal to that induced at each vortex by the sink, a criterion for jet formation can be determined. For the sake of this model, the following further assumptions will be made: 1) the cancellation of the two flows is considered only at the peak of the suction stroke, 2) the distance between the two vortices of the pair is $h$, and 3 ) the downstream position $x$ of the vortex pair at the peak of the suction stroke is the same as that found in the data of Ref. [5] which is $x / L_{o}=0.5$. The velocity induced at one vortex of the pair by the other is

$$
u_{\theta}=\frac{\Gamma}{2 \pi h},
$$

where $\Gamma$ is the circulation of each vortex. The assumption that the outflow is slug-like enables estimation of $\Gamma$ by integrating the vorticity at one side of the exit plane ejected during the blowing stroke. The vorticity in the region $d x$ below the exit plane is

$$
d \Gamma(t)=\int \frac{\partial u(t)}{\partial y} d x d y=u_{o}(t) d x
$$

and this vorticity is ejected past the exit plane during the time $d t=d x / u_{o}(t)$. Assuming a sinusoidal oscillation, the total circulation ejected per cycle is

$$
\Gamma=\int_{t=0}^{t=T / 2} d \Gamma(t)=\frac{\pi^{2}}{4} L_{o}^{2} f .
$$

Combining Eqs. (2) and (4) yields

$$
u_{\theta}=\frac{\pi L_{o}^{2} f}{8 h} .
$$

The velocity toward the exit at the location of the vortex pair and at the peak of the suction due to the sink is

$$
u_{r}=\frac{2 U_{o} h}{L_{o}}=2 f h .
$$

Equating Eqs. (5) and (6) yields the threshold stroke length for jet formation to be:

$$
\frac{L_{o}}{h}=\frac{4}{\sqrt{\pi}} .
$$

The actual dimensionless stroke necessary to form a 2-D synthetic jet is investigated using schlieren visualization and hot-wire anemometry. For nozzle widths $h=0.51,1.0,1.5$ and $2.1 \mathrm{~cm}$, the frequency is swept over the range $10 \mathrm{~Hz}<f<110 \mathrm{~Hz}$ (for the larger jet widths, some of the higher frequencies cannot be investigated due to amplitude limitations of the facility). The driver amplitude is increased from zero until a jet is detected visually. The pressure amplitude below the nozzle blocks at this amplitude and frequency is noted for each case so that it can be repeated for hot-wire measurements. A full hot-wire profile is taken at the exit plane, and these data are used to compute $L_{o} / h$ using a spatial and cycle average of the velocity data. The data are shown in 


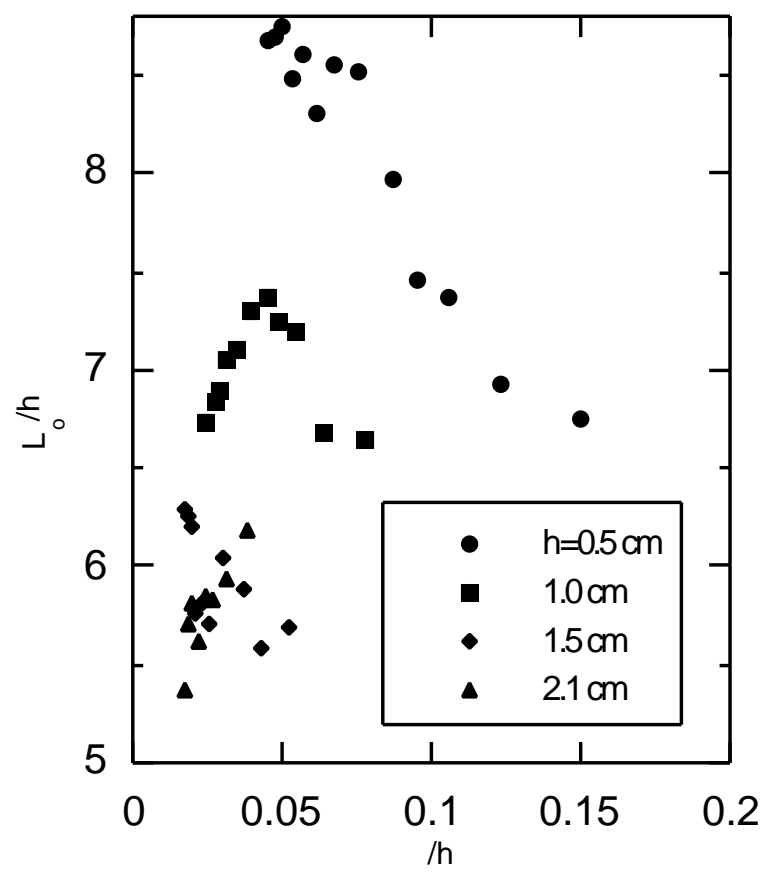

Figure 2: Map of synthetic jet formation threshold. For a given nozzle width and frequency, a synthetic jet is formed if $L_{o} / h$ is above the curve.

Fig. 2 vs the viscous penetration depth $\delta_{\nu}=\sqrt{\nu / \pi f}$ relative to the channel width.

For a given nozzle width and frequency, a jet is formed for values of $L_{o} / h$ that lie above the curve. In general, the values are larger than predicted above and larger than those for round synthetic jets $[8,11]$. Besides the obvious limitations in the model with respect to the time evolution of this process, another explanation of this discrepancy is departure from slug flow. Calculation of the circulation from the centerline velocity indicates that the actual circulation is 15-30\% greater than that obtained using a slug-flow assumption.

It is also clear that the formation threshold is not a constant, and that the variations in the threshold are larger for the smaller nozzle sizes. The reason for this is likely to be variation in the location where separation occurs and the vortex rollup begins. If the exiting flow remains attached to the exit radius $r$ over even a small distance, the effective width of the channel where the rollup begins is larger than $h$, and

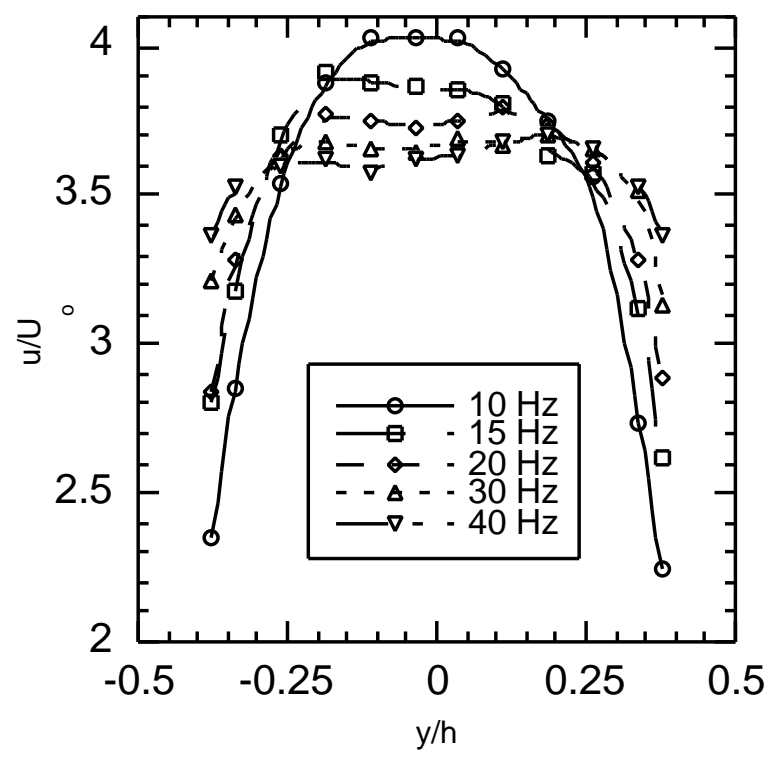

Figure 3: Profiles of velocity necessary for jet formation at $t / T=0.25$ for $h=0.51 \mathrm{~cm}$ for several frequencies ( $t=0$ is the start of the blowing stroke).

therefore the plotted value of $L_{o} / h$ is artificially high. This attachment gives the larger fractional change in effective width for the smallest $h$, where $h / r=\mathcal{O}(1)$.

Furthermore, concentrating attention on the smallest nozzle width, it stands to reason that a thinner boundary layer should remain attached longer than a thicker boundary layer [13], and therefore have a more inflated threshold. Flow visualization (not shown) confirms that higher-frequency cases separate downstream of the nozzle-lip radius. Velocity profiles at the peak of the blowing stroke $(t / T=0.25)$ are shown in Fig. 3 for $h=0.5 \mathrm{~cm}$, and it is clear that the variation in the boundary-layer thickness with frequency corresponds to the large variation in the formation threshold seen in Fig. 2. Hence, we believe that the variations seen in the threshold data are primarily due to the radius at the lip, and that, if this radius were not present, the formation threshold would be nominally constant and in the neighborhood $5.5<L_{o} / h<6.0$.

Matters are further complicated by turbulent transition in the nozzle channel or subsequent turbulent 
transition of the vortex pairs. In Fig. 4, schlieren images are shown at similar times in the stroke for $h=0.5 \mathrm{~cm}$ and $f=20 \mathrm{~Hz}$ (a) and $100 \mathrm{~Hz}(\mathrm{~b})$. It is clear that the lower frequency (and thus lower $U_{o}$ ) vortex pair is laminar, while for the higher frequency the pair is turbulent. The transition to turbulence causes a vortex pair to propagate at a lower speed [5], so transition prior to the suction stroke could influence the formation threshold.

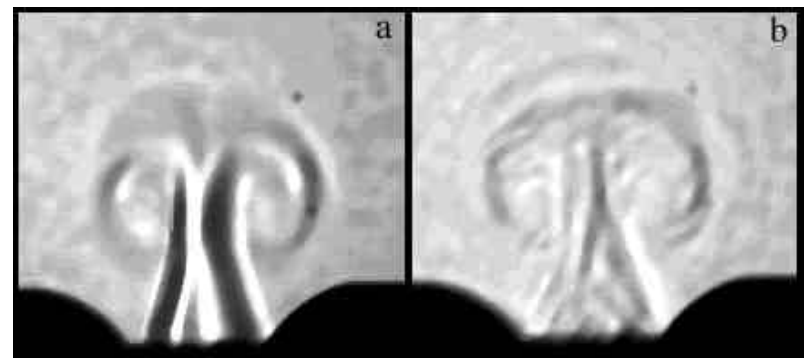

Figure 4: Schlieren images of synthetic jets at the formation threshold near the peak of the blowing stroke with $h=0.51 \mathrm{~cm}$, (a) $20 \mathrm{~Hz}$ and (b) $100 \mathrm{~Hz}$.

In addition, while our derivation of Eq. (7) assumed slug flow, different boundary-layer thicknesses will lead to different distributions of vorticity and spacing between the vortex cores. Vortex rings generated with thick boundary layers leave the exit plane earlier than those with thinner boundary layers [12].

\section{SYNTHETIC JETS AND "SIMILAR" CONTINUOUS JETS}

In this section we will compare several features of continuous jets and synthetic jets. Nine cases are studied and are summarized in the table below, with synthetic jets denoted by 'sj', an unforced continuous jet denoted by 'ufcj' and a forced continuous jet by 'fcj.'

\begin{tabular}{|rl|rrrrr|}
\hline & & $R e$ & $L_{o} / h$ & $\begin{array}{c}U \\
(\mathrm{~m} / \mathrm{s})\end{array}$ & $\begin{array}{c}f \\
(\mathrm{~Hz})\end{array}$ & $\begin{array}{c}h \\
(\mathrm{~cm})\end{array}$ \\
\hline 1 & $\mathrm{sj}$ & 2090 & 80.3 & 8.2 & 20 & 0.5 \\
2 & $\mathrm{sj}$ & 2000 & 31.0 & 7.9 & 50 & 0.5 \\
3 & sj & 2200 & 17.0 & 8.7 & 100 & 0.5 \\
4 & ufcj & 2200 & & 8.7 & & 0.5 \\
5 & fcj & 2200 & & 8.7 & 600 & 0.5 \\
6 & sj & 734 & 22.8 & 2.9 & 25 & 0.5 \\
7 & $\mathrm{sj}$ & 695 & 13.5 & 2.7 & 40 & 0.5 \\
8 & $\mathrm{sj}$ & 7500 & 18.1 & 7.4 & 20 & 2.0 \\
9 & $\mathrm{sj}$ & 14700 & 35.5 & 14.0 & 20 & 2.0 \\
\hline
\end{tabular}

The synthetic jets cover the parameter space shown in Fig. 5. The forced continuous jet is forced at the Kelvin-Helmholtz frequency of $600 \mathrm{~Hz}$, with an oscillation amplitude of $5.5 \%$ of $U_{\text {ave }}$. The jets referred to as "similar" (cases $1-5$ ) all have $R e \approx 2000$. Two synthetic jes (6-7) were made such that $u_{\max }=U_{\text {ave }}$ from the two continuous jets. Cases 8-9 will be discussed in Sec. 4.

For all of the jets with $h=0.5 \mathrm{~cm}$, in addition to exit-plane profiles and centerline data, velocity profiles are obtained in several downstream locations beyond the region where jet development occurs.

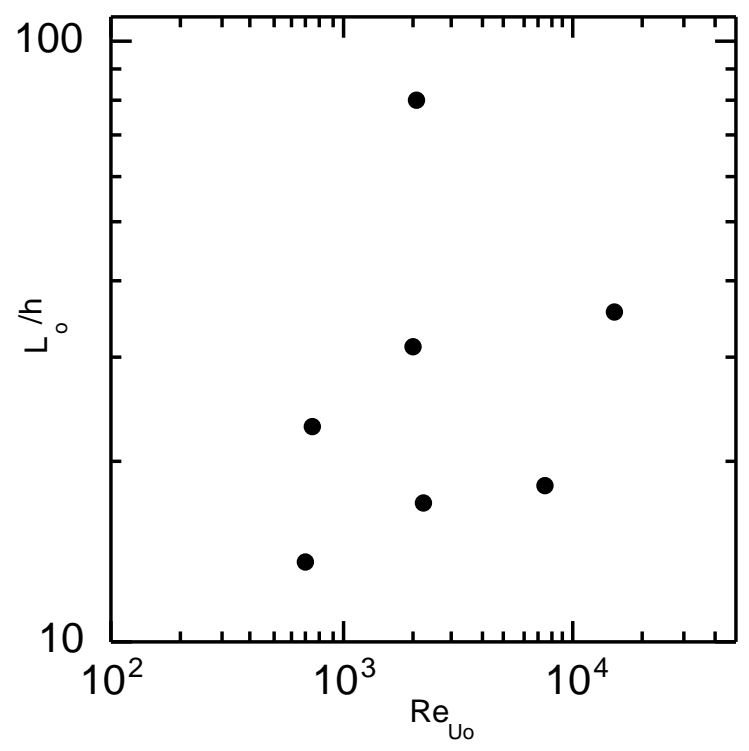

Figure 5: Parameter space for comparison of synthetic jets with various $R e_{U_{o}}$ and $L_{o} / h$. 


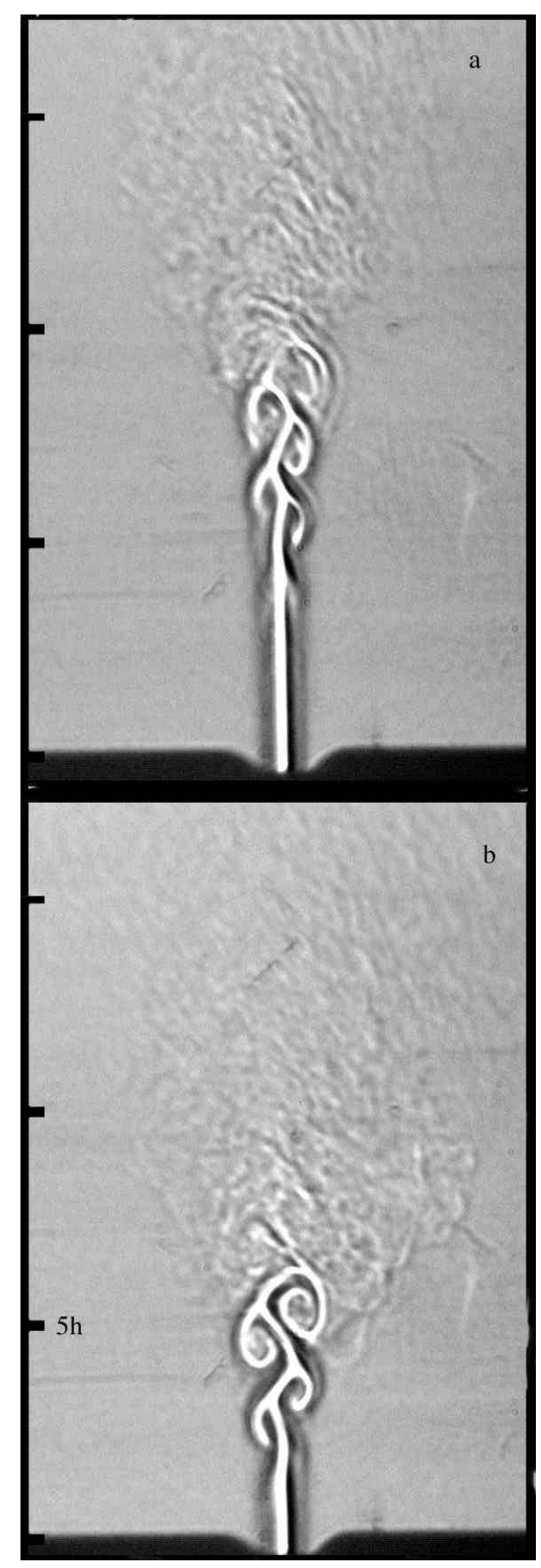

Figure 6: Schlieren images of continuous jets with $R e_{h}=2200$. (a) unforced jet, case 4 and (b) forced jet (forcing $5.5 \%$ of mean velocity, case 5 ).

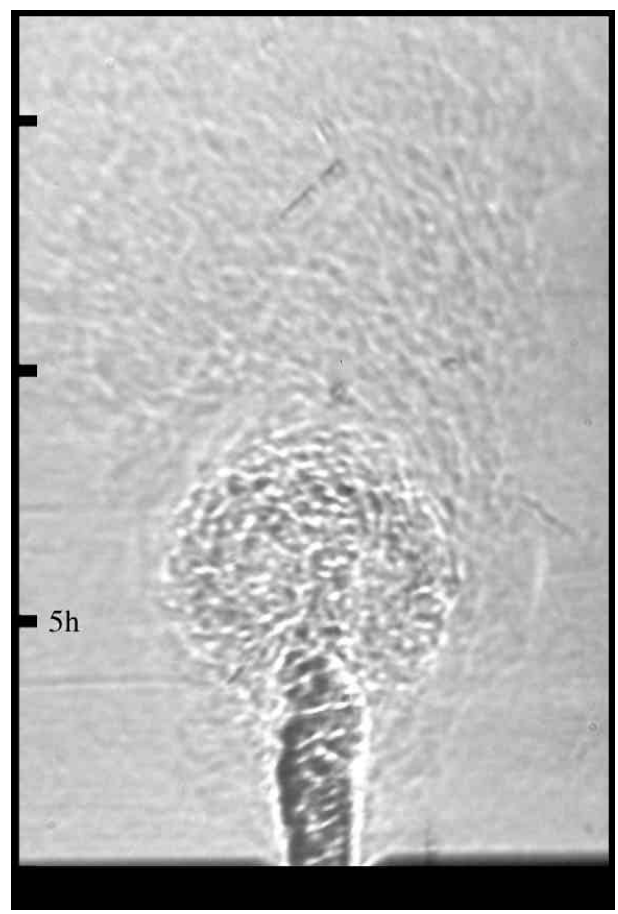

Figure 7: Schlieren image of a synthetic jet case (3) $\left(L_{o} / h=17.0\right.$ and $\left.R e_{U o}=2200\right)$ at $t / T=0.25$.

A schlieren image of the unforced continuous jet is shown in Fig. 6(a). The field of view is $9.4 \mathrm{~cm}$ by $6.1 \mathrm{~cm}$. It is clear from the image that the channel flow is laminar (as is to be expected for $R e_{h}=2200$ ). The Kelvin-Helmholtz instability results in the rollup of vortices starting at $x / h=5$, and the subsequent transition to turbulence. As shown below, the unstable band of frequencies is centered around $600 \mathrm{~Hz}$, and, when the same jet is forced at that frequency, the rollup occurs closer to the exit plane [Fig. 6(b)].

As discussed in Sec. 1, the synthetic jet has the additional parameter of $L_{o} / h$, and the features of the jet vary in time to a much larger extent than the continuous jets. The synthetic jet of case (3) is shown at $t / T=0.25$ in Fig. 7. Unlike the continuous jet, the flow exiting the nozzle appears to be turbulent. As shown below, oscillatory flows with a maximum exit velocity similar to $U_{\text {ave }}$ for the continuous jets are turbulent over much of the blowing stroke. The remnants of the turbulent vortex pair ejected during this cycle are visible at $x / h=6$, as is the ensuing 
turbulent jet downstream of this point. Comparing the photographs, it is clear that jet growth begins much closer to the exit plane for a synthetic jet than for a continuous jet, and, based on Fig. 7, it appears that the width of the jet is similar to the size of the vortex pair in the very near field.

Other distinctions can be drawn by examination of the near field $(x / h=5.9)$ power spectra for these same three jets in Fig. 8. Note that spectra (b) and (c) are displaced upward 1 and 2 decades respectively for clarity. The spectrum of the unforced continuous jet [Fig. 8(a)] has very little power except in a band near $600 \mathrm{~Hz}$, which is the KelvinHelmholtz frequency. It is clear that the continuous jet responds well to forcing at that frequency, as shown in Fig. 8(b). Fluctuations at all frequencies

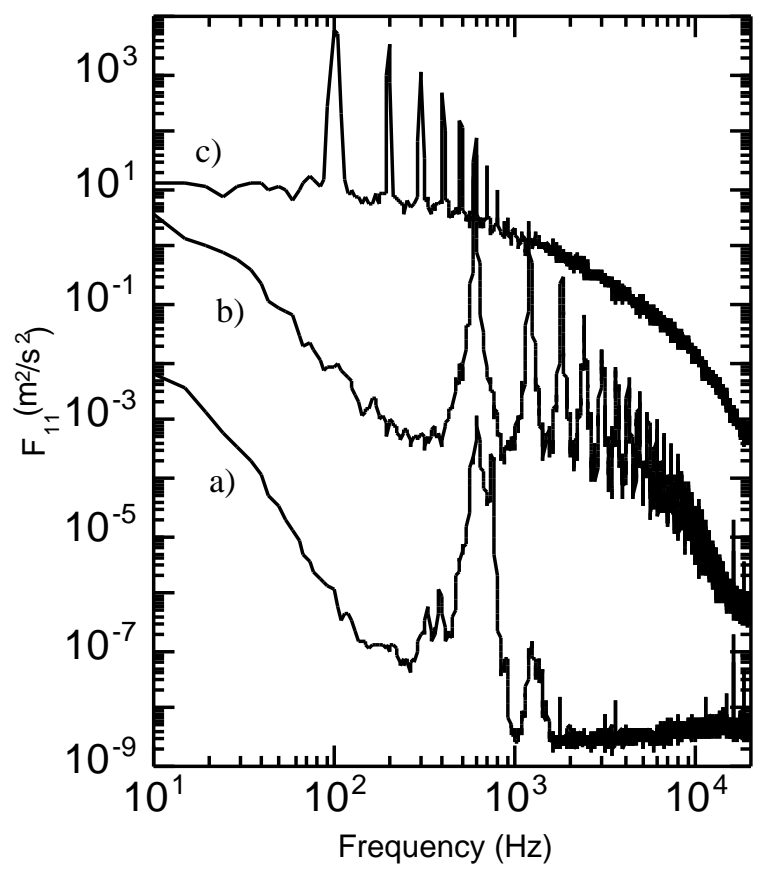

Figure 8: Velocity power spectra taken on the centerline at $x / h=5.9$ for (a) unforced continuous jet, case 4 , (b) forced continuous jet, case 5 , and (c) synthetic jet case $3\left(L_{o} / h=17.0\right.$ and $\left.R e_{U o}=2200\right)$. Note that spectra (b) and (c) are displaced upward 1 and 2 decades respectively for clarity. are higher, most notably at the forcing frequency and its harmonics. The synthetic-jet spectrum is utterly unique, looking much more like well developed turbulent flow, with most of its power at the formation frequency and its harmonics, but with large fluctuations continuing to higher frequencies.

The jets are now compared using time-averaged velocity data in the region where the jets have become self-similar. The streamwise domain of the measurements varies from case to case, since the distance over which the jet develops is a function of varied parameters such as the stroke length. The velocity profiles of each jet, normalized in the usual fashion using local values of the jet width (defined below) and the maximum time-averaged velocity $U_{c l}$, collapse for all cases as shown in Fig. 9. Each profile was measured

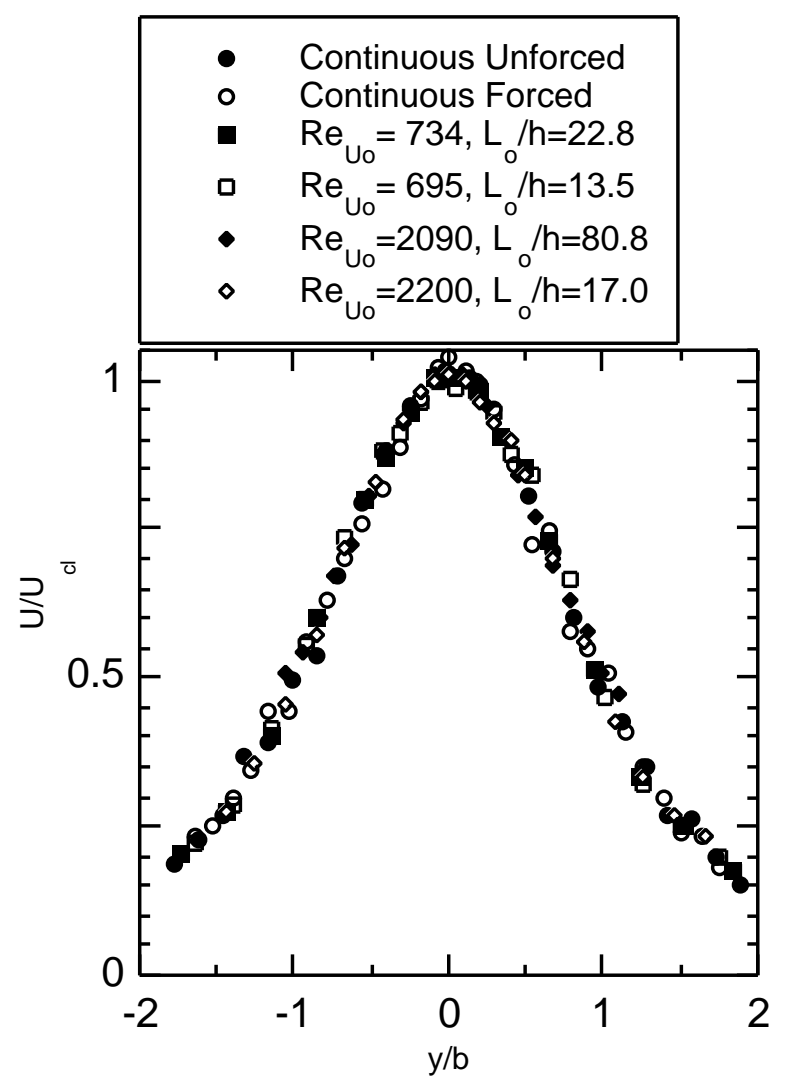

Figure 9: Mean velocity profiles in similarity coordinates. Each profile is taken at the downstream station at which $U_{c l} \approx 0.5 U_{o}$ or $0.5 U_{\text {ave }}$. 
at the downstream station at which the centerline velocity is half of $U_{\text {ave }}$ (continuous jets) or $U_{o}$ (synthetic jets). At these downstream distances, it appears that the jets have little or no memory of how they were generated. Profiles of synthetic jets with much larger or smaller $U_{o}$ values also collapse to the same shape, indicating that the use of local variables for normalization renders this measurement insensitive to $U_{o}$. Therefore, this result should not be taken as confirmation of the proposed scaling.

The cross-stream location at which the streamwise velocity is half of the centerline value is a commonly used quantitative measurement of the width of a jet. The jet width $b$ determined using the velocity profiles is plotted in Fig. 10. The width of all of the

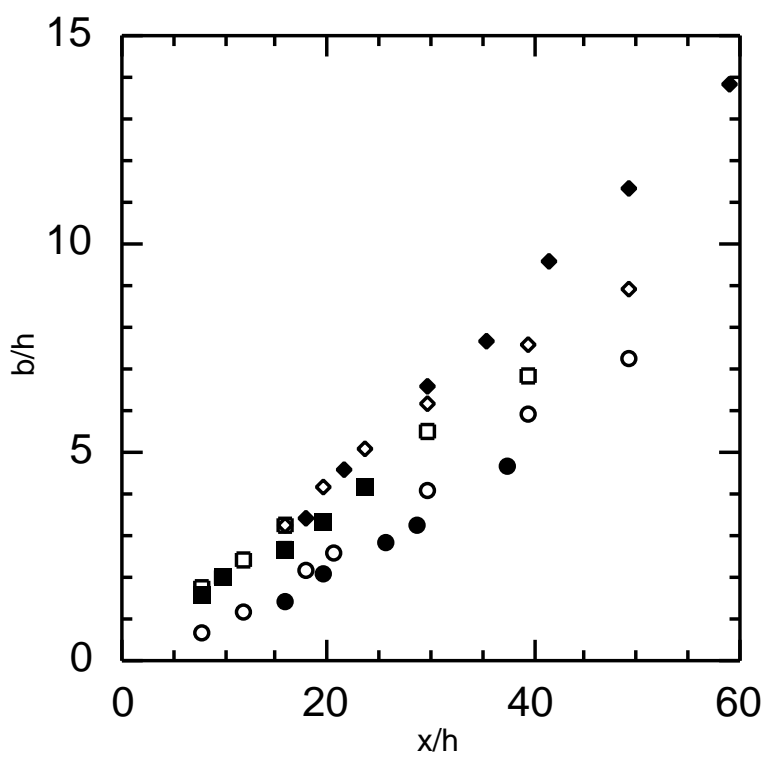

Figure 10: Width of jet based on half maximum velocity as a function of downstream distance. Symbols as in Fig. 9.

jets grows linearly with $x$ as is expected for 2-D jets [13]. The data confirm that the continuous jets are narrower near the exit plane, and the forced continuous jet is slightly wider than the unforced jet owing to increased entrainment. It also appears that $d b / d x$ is larger for the synthetic jets, especially for those with large stroke length. Also notice that the "virtual origin" of the jet, which is commonly taken to be the axial position for which $b=0$, is actually negative for the synthetic jets, with the exception of the largest-stroke-length case. The jet forms directly downstream of the vortex pair generated on the blowing stroke and therefore has a large cross-stream dimension in the near field. For continuous jets, the virtual origin tends to be facility dependent, but is positive for jets that emerge laminar. It is interesting that, in this regard, the long-stroke-length jet resembles the continuous jets. When the stroke is very long, the role of the vortex is significantly reduced, as will be shown in Sec. 4 .

The rate at which a jet widens has a direct impact on the volume flux of the jet. Since the hot-wire measurements are limited to velocities greater than $0.5 \mathrm{~m} / \mathrm{s}$ and to regions of small cross-stream velocity (compared to the local downstream velocity), the cross-stream extent of the velocity profiles is limited. To account for flux in the edges of the jet where measurements are not made, a theoretical [13] 2-D turbulent jet profile is fitted to the data at each downstream station, and this fit is integrated to obtain the volume flux as in Ref. [5]. Specifically, it is assumed that

$$
U(y)=U_{c l}\left(1-\tanh ^{2} \sigma \frac{y}{x}\right)
$$

where $U_{c l}$ and $\sigma$ are parameters of the fit. In Fig. 11, the streamwise volume flux per unit depth, $Q$, for each jet is plotted versus downstream distance. The flux values are normalized by the average volume flux at the exit, $Q_{o}=U_{o} h$. For the two continuous jets, the flow rate increases linearly with downstream distance, and is nearly identical for both cases. The synthetic-jet behavior is more complicated. The synthetic-jet curves lie above the continuous-jet values at all downstream stations, because the rollup of the synthetic-jet vortex pair entrains much more fluid than does the laminar continuous-jet column. For most of the synthetic jets, it appears that the initial rate of increase in the volume flux with downstream distance is much larger than for the continuous jets, and that far enough downstream $d Q / d x$ levels off to a value closer to the continuous jets.

The fact that the forced jet is wider while its volume flux is identical to that of the unforced jet can be explained by the behavior of the centerline mean velocity shown in Fig. 12. The velocity begins to decrease from the exit value after the jet becomes turbulent near $x / h=5$ for the forced jet, while this 


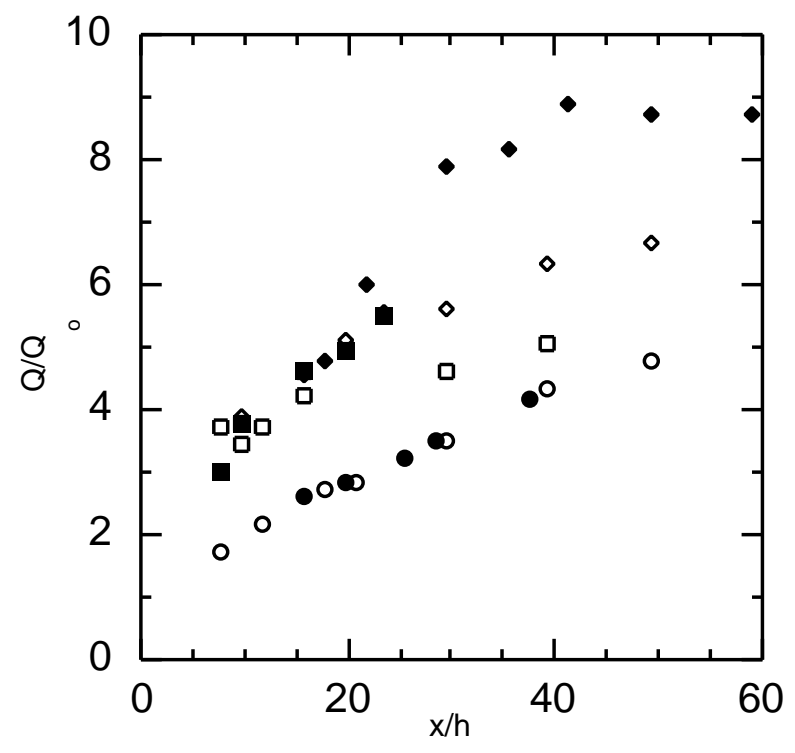

Figure 11: Streamwise volume flux per unit depth as a function of downstream distance. Volume flux is normalized by $U_{o} h$ for the synthetic jets and by $U_{\text {ave }} h$ for the continuous jets. Symbols as in Fig. 9 .

does not occur until $x / h=10$ for the unforced jet. Therefore, the forcing results in a jet that is wider and slower, but of the same volume flux.

The mean velocity at the exit is zero, and rises $[5,11]$ to a level very near $U_{o}$ for 2 -D synthetic jets (the value is higher for round synthetic jets [11]) before the $-1 / 2$ power-law decay typical of plane jets begins. Despite the range of Reynolds number and dimensionless stroke length in the data of Fig. 12, the centerline-velocity behavior is very similar in every case. In the near field, the synthetic jets consistently lie below the continuous jets, indicating that the synthetic jets are consistently wider and slower than similar continuous jets.

Another parameter that can be compared is the momentum flux of the jet. In applications where momentum interactions between a synthetic jet actuator and a primary jet are important [4], this is the most relevant parameter. The momentum flux per unit depth $J$ is computed from the profile data in a manner similar to that for the volume flux, and the results are shown in Fig. 13. It is a common assumption for continuous jets that the momentum flux is

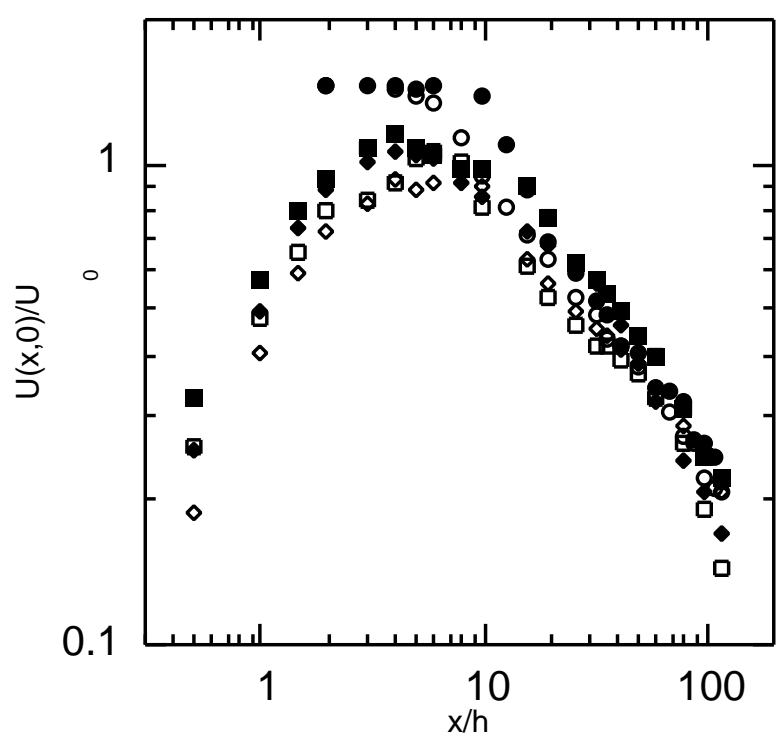

Figure 12: Time-averaged centerline velocity versus downstream distance. Symbols as in Fig. 9.

invariant with downstream distance (assuming zero pressure gradient and a jet emanating from an exit plane with walls normal to the direction of the flow). The present data indicate that the unforced continuous jet has larger momentum flux than the forced jet (this is consistent with a thinner, faster jet), and that $J$ initially decays for both continuous jets. It is unlikely that this decay is real, and more likely that the fitting procedure described above is not valid at the more near-field values of $x$, which may indicate that the mean profiles have not yet reached a fully self-similar state. However, for both continuous jets, the momentum flux does eventually become a constant near unity (closer to the exit plane for the forced jet than the unforced jet). The normalized momentum flux of the synthetic jets is larger in general than that of the continuous jets. Similar to Ref. [11] where no fitting was used to compute momentum flux from PIV velocity data, the synthetic-jet momentum flux decreases with downstream distance and the jets with larger $L_{o} / h$ result in larger final momentum flux, since less (or likely none) of the fluid ejected during the blowing stroke is reingested during the suction stroke.

It should be noted that the actual momentum flux 


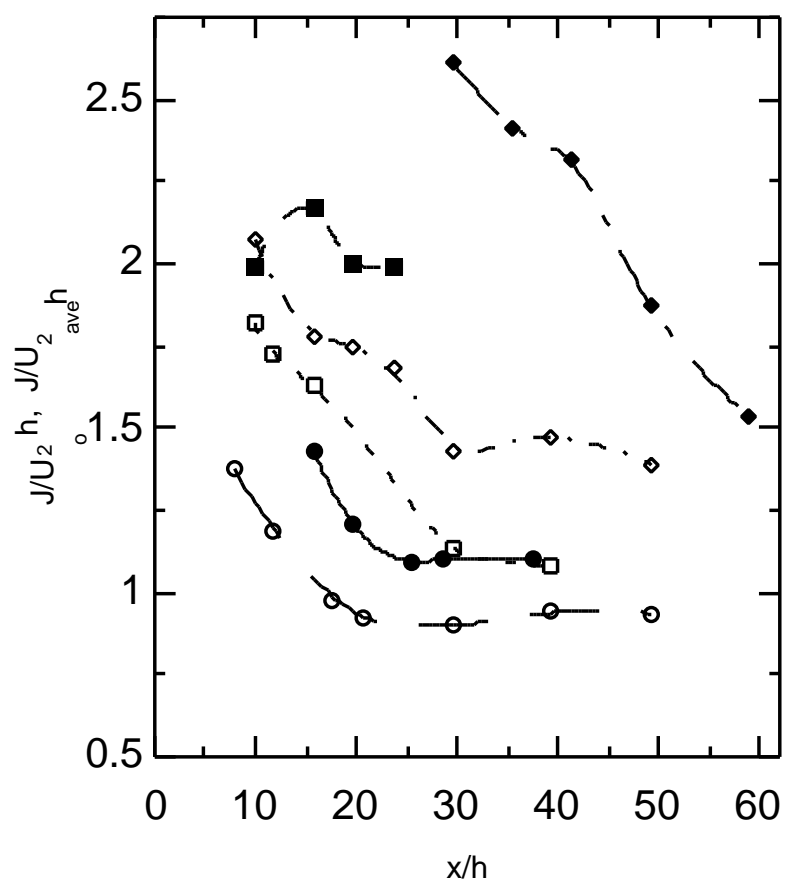

Figure 13: Dimensionless momentum flux as a function of downstream distance. Momentum flux is normalized by $U_{o}^{2} h$ for the synthetic jets and by $U_{\text {ave }}^{2} h$ for the continuous jets. Symbols as in Fig. 9.

out of the synthetic jet nozzle is not $U_{o}^{2} h$ as is implied by the normalization scheme used here. This normalization fails to account for 1) departure from slug flow profiles that will increase the momentum flux for a given average velocity (this is also true for the continuous jets), 2) departures from sinusoidal oscillations which can also increase the momentum flux for a fixed $U_{o}$, and most importantly 3 ) the momentum flux of the entire suction stoke, which counterintuitively has the same sign as the flux of the blowing stroke.

It is desirable to compare the jets using a measure that is not sensitive to their shape, and power spectra of the streamwise velocity component provide such a measure. In Fig. 14, power spectra taken at $x / h=30$ for all nine cases (including two discussed more extensively in Sec. 4, which have $h=2.03$ ) are plotted. All of the spectra are smoothed to make them more easily distinguishable, and the numbers in parentheses in the figure legends refer to the case number. The
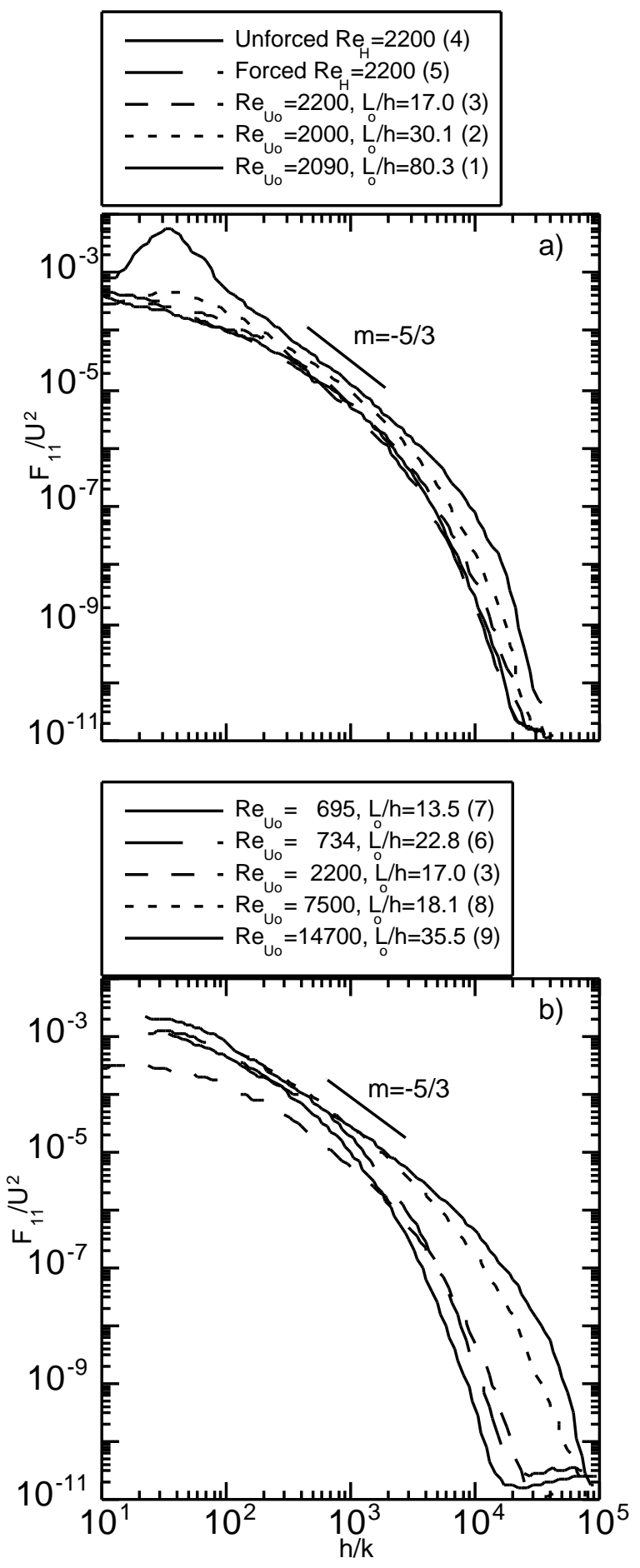

Figure 14: Power spectra taken on the jet centerline at $x / h=30$. Numbers refer to the jet cases. (a) jets with similar Reynolds numbers and (b) jets with similar stroke lengths. 
power levels are normalized by the square of the average orifice velocity $\left(U_{o}\right.$ or $\left.\mathrm{U}_{\text {ave }}\right)$, are plotted against the inverse of the scale $k=U_{c l} / f$ relative to the orifice size. The flow scale is calculated by invoking Taylor's hypothesis of frozen turbulence, and using the local mean velocity. The five "similar" jets [Fig. 14 (a)] have similar spectra at this location (and indeed at all locations downstream of the development of the jets), although the effect of the stroke length can clearly be seen. The shortest stroke length synthetic jet (case 3) matches the continuous jets nearly identically. As the stroke length is increased, the spectrum gains more power, and extends to smaller scales. For the longest stroke length (case 1), the measurement station is within the region in which the flow is highly oscillatory, and therefore a peak at the forcing frequency can be seen at $h / k=30$. It is likely that the frozen turbulence assumption is not valid here, which may explain the stroke length effect.

The effect of the Reynolds number can be seen in Fig. 14(b) in the extension of the inertial subrange of scales, indicated by a $-5 / 3$ power-law behavior, from $h / k=2000$ for $R e \approx 2000$ to 6000 for $R e=$ 14700. For jets with $R e \approx 700$, an inertial subrange is difficult to detect. A larger inertial subrange also leads to power at smaller scales, as is to be expected in any turbulent flow.

We have seen that the appropriateness of our choice of $U_{o}$ as a velocity scale depends on which dependent variable is examined. "Similar" synthetic jets are slower and wider and have larger momentum flux than continuous jets do, if normalized using the $U_{o}$ scale. Based on these results, it is clear that no single choice of velocity scale would result in properly scaled data for all of the aforementioned parameters.

\section{EFFECTS OF DIMENSION- LESS STROKE LENGTH AND REYNOLDS NUM- BER}

In this section, we will emphasize the role played by the synthetic jet Reynolds number $R e_{U_{o}}$ and the dimensionless stroke length $L_{o} / h$. In addition to the data above, data from two more synthetic jets with a larger nozzle $(h=2.0 \mathrm{~cm})$ will be examined.
First, we compare schlieren photos taken at the peak of the blowing stroke for two jets with similar stroke lengths, but disparate Reynolds numbers. The synthetic jet in Fig. 15(a) has a similar dimensionless stroke length (13.5) to the one pictured in Fig. 7, while $R e_{U_{o}}$ is much lower (695). The vortexpair structure looks very similar in size and shape. However, careful examination (a moving picture may be required) reveals smaller-scale structure in the vortex, the stem, and the ensuing jet for the jet with the higher Reynolds number. A synthetic jet with a Reynolds number nominally matched to the one in Fig. 7 but with a stroke nearly five times larger is shown in Fig. 15(b). Since the vortex pairs' position at a given time scales on $L_{o} / h$, as shown below in the discussion of Fig. 17, the pair has moved out of the visualization domain long before the image was acquired. For dimensionless stroke lengths $\mathcal{O}(100)$, the vortex pair grows very rapidly and is difficult to detect in the images, perhaps because of increased mixing with the downstream fluid.

It should be noted that in each of these cases the vortex pair appears to be turbulent at all times, in contrast to the results of Ref. [5] in which the pairs were initially laminar and consistently transitioned to turbulence when the suction stroke began.

The role of the stroke length, which is clear from the flow visualization, is also captured in phaselocked velocity data on the centerline of the jet. Traces of the streamwise velocity for the smallestand largest-dimensionless-stroke-length jets (7 and 1) $\left(L_{o} / h=13.5\right.$ and 80.8 respectively) are shown in Fig. 16. The stroke length of case 7 is similar to that reported in Ref. [5] $\left(L_{o} / h=20\right)$, and the resulting centerline traces are also similar, except for the absence of a small peak leading the maximum velocity (this peak is present in the current data for a jet with $L_{o} / h \approx 20$, not shown). The peaks in the velocity traces correspond to the arrival time of the center of the vortex pair at each station. The centerline velocity peak initially increases above the exit-plane value, and the duration and magnitude of the backward flow decrease rapidly with downstream distance. For the larger stroke length, the traces are altered considerably. The vortex pair is manifested by a secondary peak trailing the maximum velocity. The magnitude of the velocity peaks decreases much more rapidly with downstream distance than in the shorter-stroke 


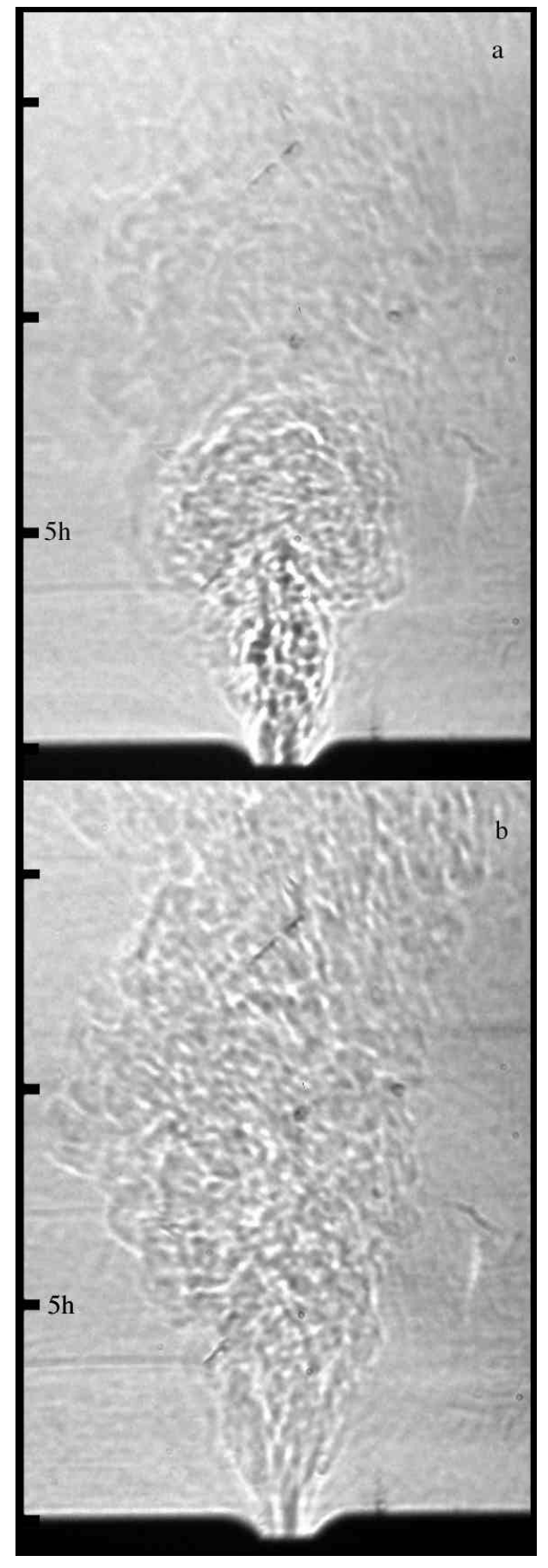

Figure 15: Schlieren images of synthetic jets at $t / T=$ 0.25 with (a) $L_{o} / h=13.5$ and $R e_{U o}=695$ (case 7) and (b) $L_{o} / h=80.8$ and $R e_{U o}=2090$ (case 1).
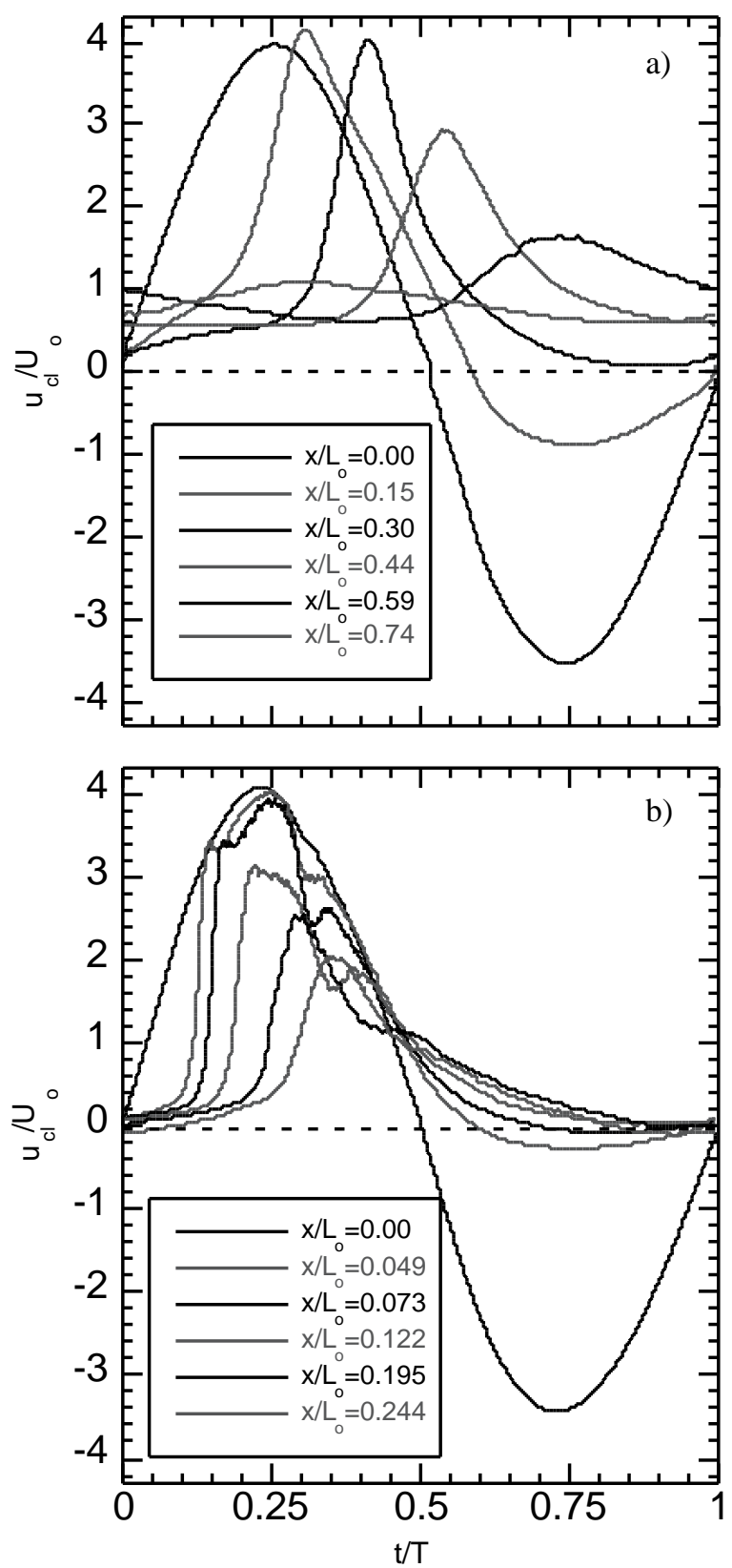

Figure 16: Centerline velocity traces for (a) $R e_{U_{o}}=$ $695, L_{o} / h=13.5$ (case 7) and (b) $R e_{U_{o}}=2090$, $L_{o} / h=80.8$ (case 1$)$. 
jet. The fluctuations during the blowing are large enough that the 130 cycles over which the data were phase averaged are not sufficient for convergence to a smooth curve. Also notice that very little reverse flow is observed beyond the exit plane.

These traces can be used to infer the arrival time of the vortex pair at each downstream station as was done in Ref. [5]. Vortex trajectories are plotted in Fig. 17 along with the largest and smallest Reynolds number cases from Ref. [5]. No clear trend with $R e_{U_{o}}$ or $L_{o} / h$ can be found, and the data from Ref. [5] lie in the middle of the present data. It is likely that differences in trajectory come from the turbulent transition of the vortex pairs occurring at different times. These transitions can be due to core instabilities that do not necessarily scale with either of the parameters used here.

Although it is difficult to detect in the flow visualization, an important effect of Reynolds number on a synthetic jet is the impact on the exit plane velocity profiles, which in turn strongly affect the behavior of the vortex pairs generated during each stroke. As discussed in Ref. [10], the turbulent transition in oscillating flow is more complicated than in steady flow. The addition of the frequency parameter to the problem creates an additional dimensionless parameter that governs transition. Most workers in oscillatory flow transition use the parameters $R e_{\max }=U_{\max } h / \nu=\pi U_{o} h / \nu$ and $h / \delta_{\nu}$. Although different workers have published various transition criteria, it seems clear that oscillating pipe flow becomes turbulent if $R e_{\max } \gtrsim 600 h / \delta_{\nu}$.

In Fig. 18, phase-averaged velocity profiles as well as fluctuation profiles at the exit plane at five equal increments in phase during the acceleration part of the blowing stroke are shown for four of the cases from Fig. 5. Two of these jets share the frequency of $20 \mathrm{~Hz}$ (which for a laminar case would dictate the boundary layer thickness) and two have similar values of $U_{o}$, while two nozzle widths and three distinct values of $R e_{U_{o}}$ and $L_{o} / h$ are represented. Cases with large nozzle boundary layers, such as in Fig. 18(c), require a larger centerline velocity to achieve a fixed $U_{o}$. It is clear that the exiting flow is not laminar in all of these cases, since laminar oscillating pipe flow would have peaks near the sides of the channel and lower rms levels. The rms levels in Figs. 18(a-d) all have features indicating that the flow is at least "weakly

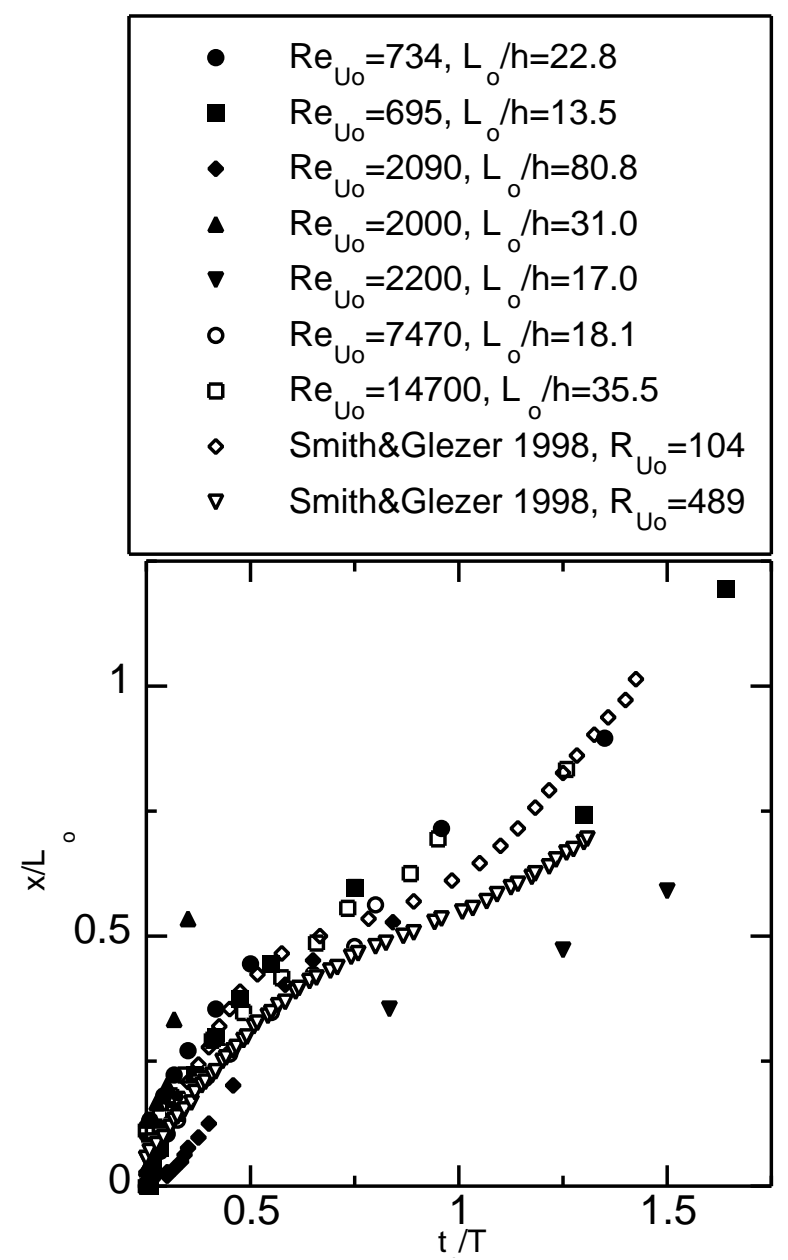

Figure 17: Trajectories of the vortex pairs. Two cases from Ref. [5] are included for reference.

turbulent" and in some cases "conditional turbulent" in the parlance of Ref. [10]. The conditionally turbulent transition is marked by a sudden increase in the fluctuation levels, especially in the boundary layers, as the acceleration phase ends. This is certainly the case in Figs. 18(c-d), were the rms levels increase rapidly as the phase approaches $t / T=0.25$. In contrast, the higher frequency case 3 in Fig. 18(b) has a larger value of $h / \delta_{\nu}$ and is therefore less prone to transition. The rms levels in this case increase steadily with velocity, which is more indicative of the "weakly turbulent" regime. 

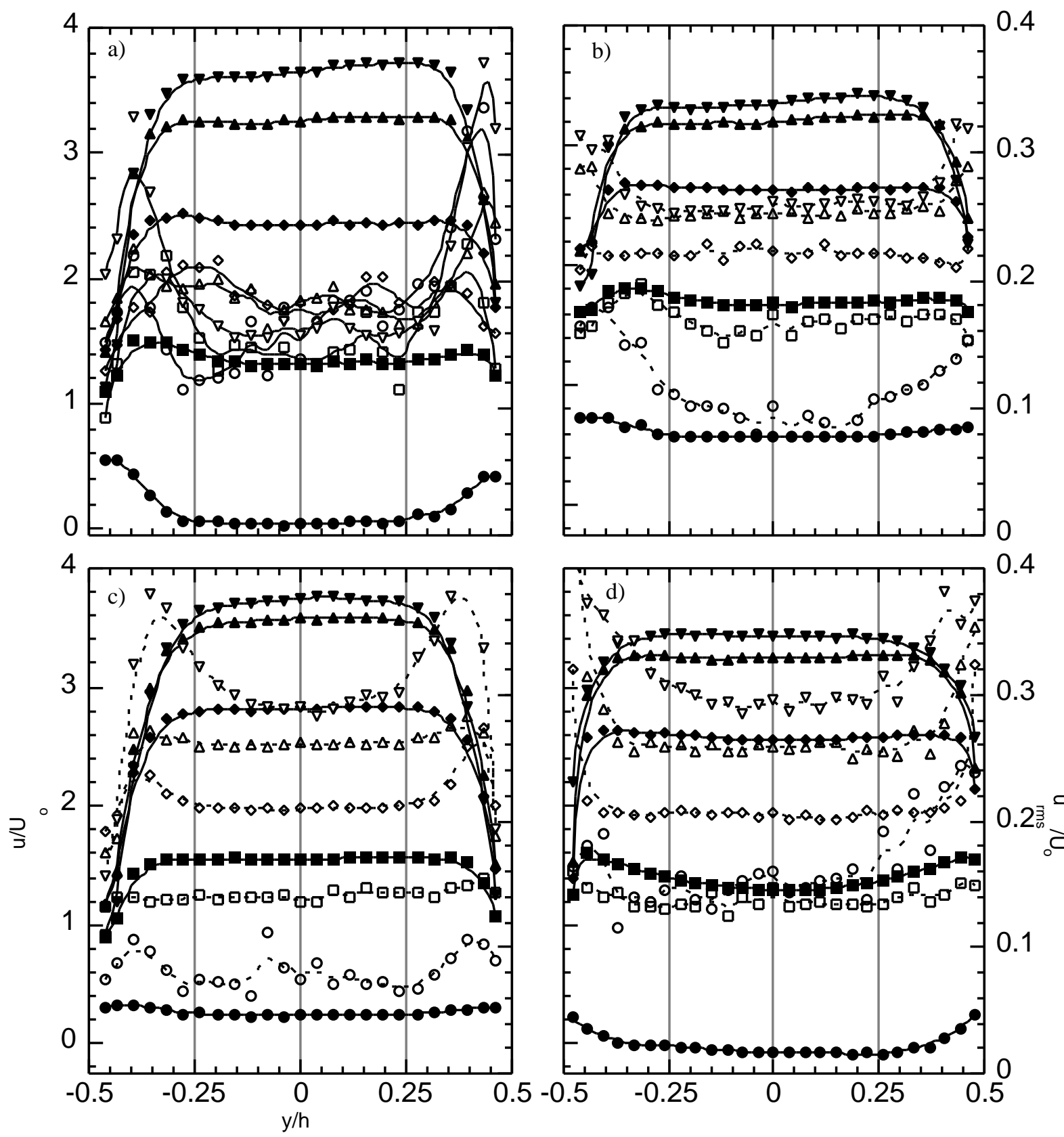

Figure 18: Streamwise velocity profiles at five equal phase increments during the acceleration part of the blowing stroke. $\bullet t / T=0.0, \boldsymbol{\square} t / T=0.06, \forall t / T=0.12, \boldsymbol{\Delta} t / T=0.18, \boldsymbol{\nabla} t / T=0.24$. Closed symbols are phase-averaged velocity, open symbols are corresponding rms fluctuations. (a) case $7 R e_{U_{o}}=695, L_{o} / h=$ 13.5, (b) case $3 R e_{U_{o}}=2200, L_{o} / h=17.0$, (c) case $1 R e_{U_{o}}=2090, L_{o} / h=80.3$, and (d) case $9 R e_{U_{o}}=$ $14700, L_{o} / h=35.5$. 

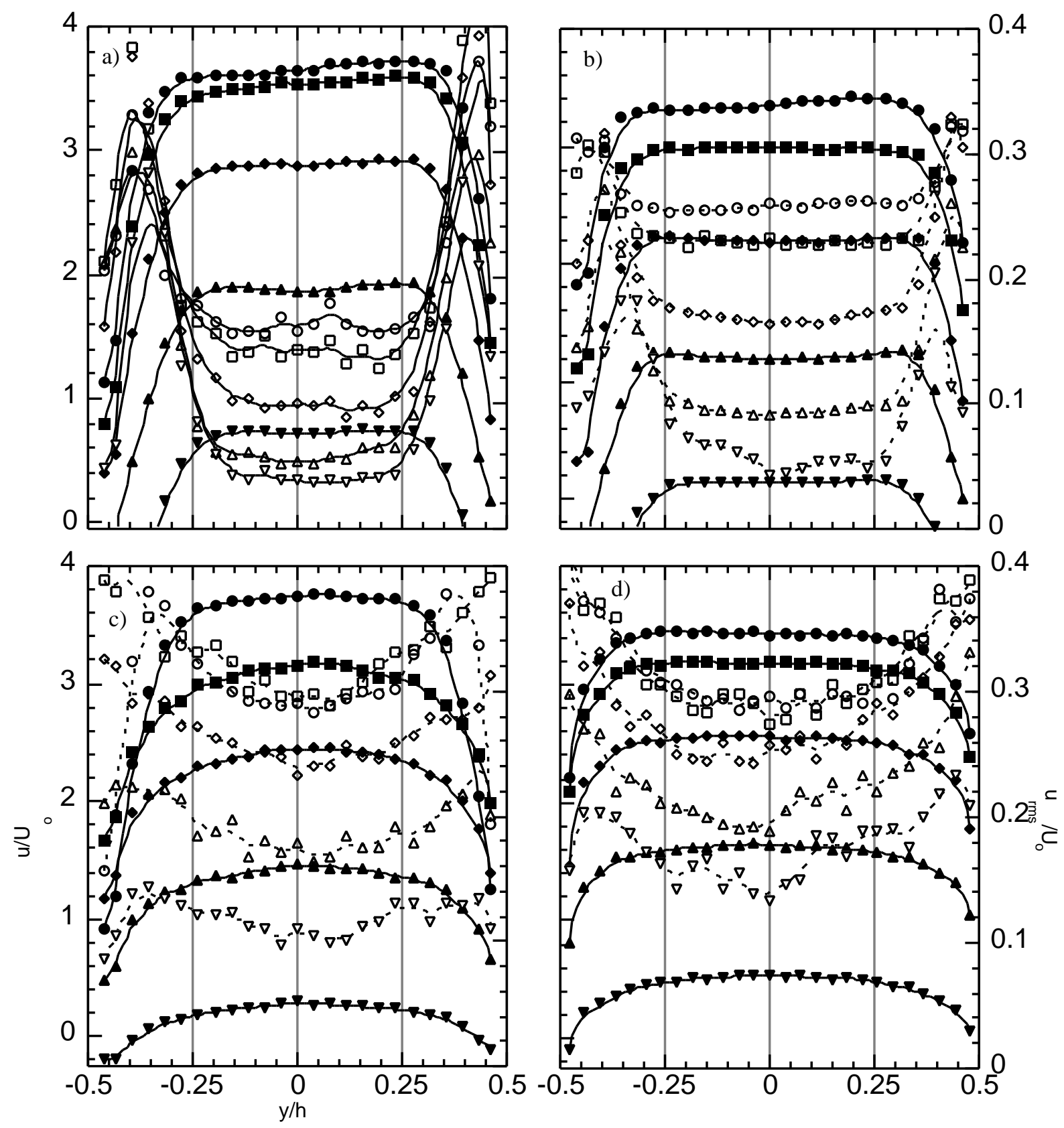

Figure 19: Streamwise velocity profiles at five equal phase increments during the deceleration part of the blowing stroke $\bullet t / T=0.24, \boldsymbol{\square} t / T=0.30, \forall t / T=0.36, \boldsymbol{\Delta} t / T=0.42, \boldsymbol{\nabla} t / T=0.48$. Closed symbols are phase-averaged velocity, open symbols are corresponding rms fluctuations. (a) case $7 R e_{U_{o}}=695, L_{o} / h=$ 13.5, (b) case $3 R e_{U_{o}}=2200, L_{o} / h=17.0$, (c) case $1 R e_{U_{o}}=2090, L_{o} / h=80.3$, and (d) case $9 R e_{U_{o}}=$ $14700, L_{o} / h=35.5$. 


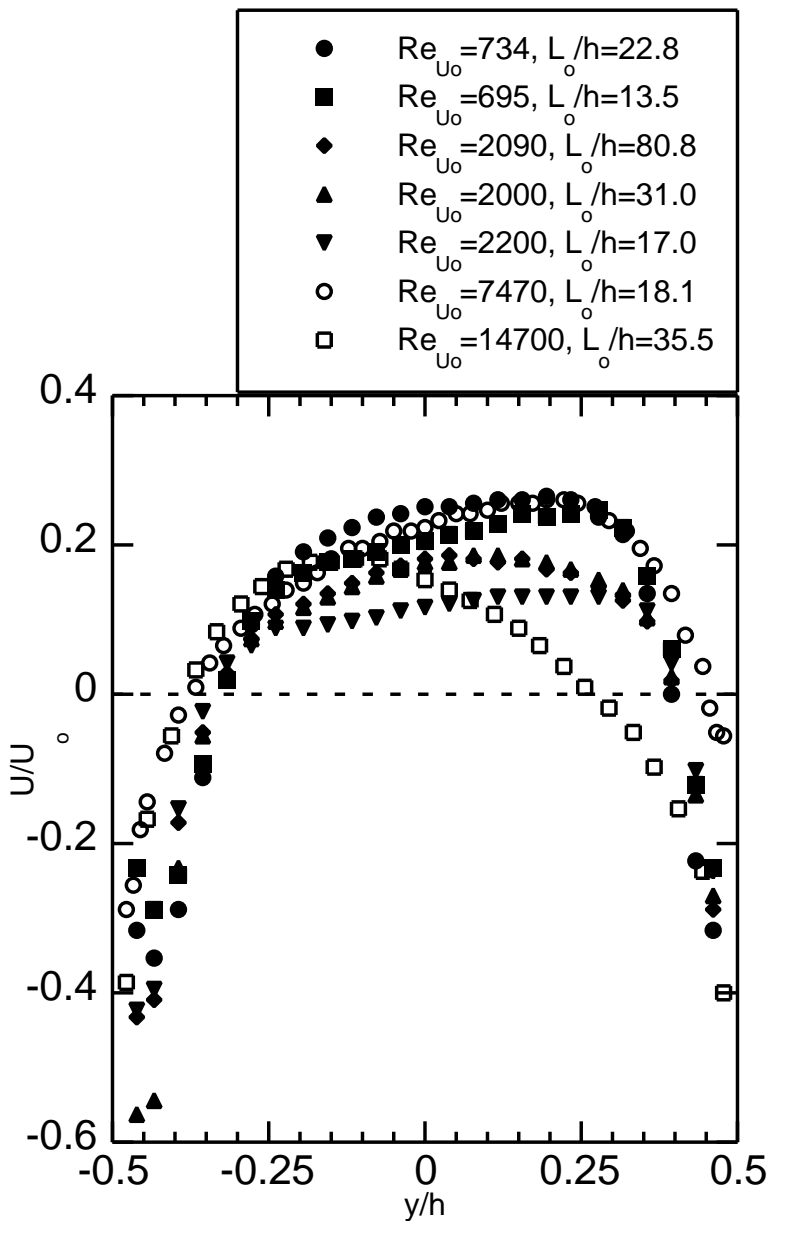

Figure 20: Time-averaged velocity at the exit plane for various synthetic jets.

The very short stroke length case 7 in Fig. 18(a) has fluctuations in the core of the flow that vary little with phase during the acceleration. It is likely that this "turbulence" was generated during the previous blowing stroke, was ingested, and is now being expelled. For this and perhaps other reasons, it appears that the oscillating flow at the exit of a synthetic jet is less stable than purely oscillatory pipe flow (the values of $R e_{\max } \delta_{\nu} / h$ range from 330 to 800 for the jets described above) .

Profiles during the deceleration part of the blowing stroke are shown in Fig. 19. The fluctuation levels of the conditionally turbulent cases identified above either continue to increase or become constant as the declaration begins. Also notice that the cross-stream extent of the region of large fluctuations grows as the blowing stroke ends, and that this growth is accompanied by an increase in the boundary-layer thickness. The weakly turbulent jets (cases 7 and 3) [Fig. 19 (ab)] are similar to laminar oscillating pipe flow in that the boundary layers begin to lead the core flow in phase considerably, crossing zero before $t / T=0.42$.

While boundary layers are formed inside of the channel on the outstroke, the inward stroke at the exit plane is entry flow, and therefore has very thin boundary layers. As a result of the asymmetry between the blowing and suction boundary-layer thicknesses, a cross-stream distribution of time-average flow develops, with positive flow in the core and reverse flow at the sides as seen in Fig. 20. In many of the cases, the mean flow is not symmetric about $y=0$, and the profile tilts to one side or the other, indicating that the mean profiles are very sensitive to asymmetries in factors such as geometry and transition. The time-averaged velocity scales on $U_{o}$, and is a strong function of the boundary-layer thickness (relative to $h$ ).

For synthetic-jet-flow-control applications in which the actuator is used to inject a flow scale into the base flow, it is crucial to know the streamwise distance over which one can expect this scale to persist. We investigate this matter, and how it is affected by the same two parameters, $R e_{U_{o}}$ and $L_{o} / h$, by examining the power spectrum of the streamwise velocity component as a function of downstream distance. Specifically, the component of the spectrum at the forcing frequency, $A_{f}$, which is indicative of the influence of the forcing, is plotted versus downstream distance for all seven synthetic jets in Fig. 21.

The spectral power is normalized by the square of $U_{o}$, while the streamwise distance is normalized by $h$. It should be noted that the values of $A_{f}$ are arbitrary due to windowing in the FFT procedure, and no meaning should be placed on them. The relative levels between cases are significant, however, and the data reveal that they initially scale on $U_{o}$. For some of the jets, the levels are initially increasing with downstream distance, which is due to rectification of the velocity signals causing power at the fundamental to appear at the second harmonic. As the vortex pair diffuses due to viscosity and turbulent fluctuations, the power at the forcing frequency decreases. The breakdown of the vortex pair is indicated by an 


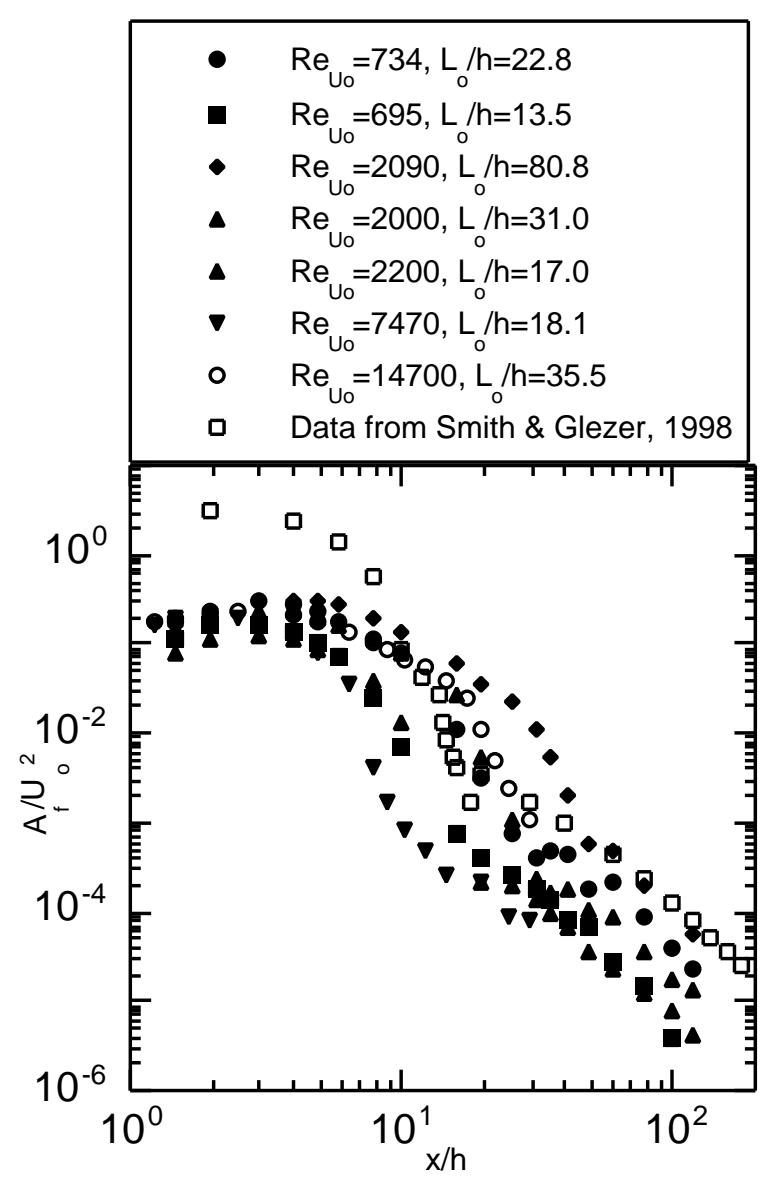

Figure 21: Normalized spectral component at the driving frequency as a function of downstream distance. One case from Ref. [5] is included.

abrupt change in the slope of the curve, which occurs consistently near $x / L_{o}=1$. At this location, the normalized value of $A_{f}$ is generally two decades down from the initial value. Downstream of this position, the spectral decay follows a -2 power law, which is consistent with the sensor measuring the sound wave generated by the synthetic jet.

Similar results were reported in Ref. [5] for a 2D synthetic jet with $R e_{U_{o}}=383$ and $L_{o} / h=20.2$ and are shown in Fig. 21. In general, these lowerReynolds-number results are very similar, except that it appears that the difference between the initial level and that at the point where the vortex breaks down is closer to three decades in Ref. [5].

\section{CONCLUSIONS}

A minimum stroke length exists below which no synthetic jet is formed. As $L_{o} / h$ is increased beyond this level, the jet momentum is increased, as the vortex pair escapes the influence of the suction stroke. If $L_{o} / h$ is further increased, the rollup of the vortex pair is altered, although the alteration seems to have no impact on the trajectory data. Synthetic jets with large stroke lengths have more small scale motions than similar Reynolds-number-jets with the smaller stroke lengths.

In the far-field, a synthetic jet bears much resemblance to a continuous jet. However, in the near-field, a synthetic jet entrains more fluid and thus grows faster than a continuous jet. The effect of $R e_{U_{o}}$ is seen in the turbulent transition of the flow exiting the nozzle, the transition of the vortex pair, and the turbulent characteristics of the developed jet flow.

In general, the finding from Refs. [5] and [11] that the near-field evolution of the synthetic jet is dictated by $L_{o} / h$ has been extended to Reynolds numbers near 15000 and dimensionless stroke lengths greater than 80. However, contrary to Refs. [5] and [11], the far field behavior of synthetic jets appears to be a function of both $R e_{U_{o}}$ and $L_{o} / h$.

\section{ACKNOWLEDGEMENTS}

The authors acknowledge Chris Espinoza and Ronald Haggard for their skill in the construction of the facility used in this study, and David Gardner for considerable help with the phase-locking electronics. Thanks also go to James Allen for many helpful conversations on jet formation. We would also like to acknowledge the financial support of the Los Alamos National Laboratory LDRD funds.

\section{References}

[1] B. L. Smith and A. Glezer. Vectoring of a high aspect ratio rectangular air jet using a zero-netmass-flux control jet. Bull. Am. Phys. Soc. 39, 1994.

[2] M. Amitay, A. M. Honohan, and A. Glezer. Aerodynamic control using synthetic jets. AIAA Fluids 2000 meeting, 2000. Paper 2000-2401. 
[3] B. L. Smith and A. Glezer. Jet vectoring using synthetic jets. Submitted to J. Fluid Mech., 2001.

[4] B. L. Smith and A. Glezer. Vectoring and smallscale motions effected in free shear flows using synthetic jet actuators. AIAA 35th Aerospace Sciences Meeting, 1997. Paper 970213.

[5] B. L. Smith and A. Glezer. The formation and evolution of synthetic jets. Phys. Fluids, 10(9):2281-2297, 1998.

[6] D. P. Rizzetta, M. R. Visbal, and M. J. Stanek. Numerical investigation of synthetic jet flowfields. AIAA Journal, 37(8):919-927, 1999.

[7] C. Y. Lee and D. B. Goldstein. Two-dimensional synthetic jet simulation. AIAA 38th Aerospace Sciences Meeting, 2000. Paper 2000-0406.

[8] U. Ingard. On the theory and design of acoustic resonators. J. Acoust. Soc. Am., 25(6):10371061, 1953.

[9] B. L. Smith and G. W. Swift. Measuring secondorder time-averaged pressure. To appear in $J$. Acoust. Soc. Am., 2001.

[10] M. Ohmi, M. Iguchi, K. Kakehashi, and M. Tetsuya. Transition to turbulence and velocity distribution in an oscillating pipe flow. Bulletin of the JSME, 25:365-371, 1982.

[11] B. L. Smith, M. A. Trautman, and A. Glezer. Controlled interactions of adjacent synthetic jets. AIAA 37th Aerospace Sciences Meeting, 1999. Paper 99-0669.

[12] W. Zhao, S. H. Frankel, and L. G. Mongeau. Effects of trailing jet instability on vortex ring formation. Phys. Fluids, 12(3):589-596, 2000.

[13] H. Schlichting. Boundary-Layer Theory. McGraw Hill, 1968. 